\title{
Tratamiento secuencial con tubo de Montgomery y stent de Dumon en estenosis post-intubación. Una opción efectiva y segura cuando el tratamiento quirúrgico no es una alternativa
}

\author{
Sequential treatment with Montgomery tube and Dumon \\ stent in postintubation stenosis. An effective and safe \\ option when surgery is not possible
}

\author{
Jaime Alberto Barreto Menéndez ${ }^{(1)}$, Carlos Ernesto Garavito Barandica ${ }^{(2)}$, Reynaldo Plata \\ VALDIVIESO ${ }^{(3)}$, BIBIANA MEJÍA CÁRDENAS ${ }^{(4)}$
}

\section{Resumen}

Los stent son dispositivos que se implantan en la tráquea, la carina o los bronquios principales con la finalidad de proporcionar un soporte que permita mantener la permeabilidad de estas estructuras. Se distinguen dos grupos principales de acuerdo con su constitución: metálicos y de silicona. Cada grupo tiene propiedades e indicaciones particulares que deben considerarse siempre en cada paciente. Los stent metálicos constituyen una opción muy atractiva debido a la relativa facilidad de colocación, lo que ha contribuido a incrementar su uso en los últimos años. La experiencia acumulada en tales intervenciones, ha permitido identificar una gran cantidad de complicaciones a tener en cuenta, sobre todo en quienes presentan estenosis de la vía aérea de etiología no maligna. En el mundo, esta serie de observaciones ya ha generado determinaciones por parte de entes gubernamentales, como es el caso de la Administración de drogas y Alimentos (FDA, su sigla en inglés), que ha recomendado no usar stent metálicos, recubiertos y no recubiertos en patologías de tipo benigno. Se expone el caso de un paciente de 59 años con estenosis traqueal post intubación quien fue tratado en forma exitosa, inicialmente con tubo de Montgomery y luego con stent de Dumon.

Palabras clave: estenosis traqueal, stent de Dumont, tubo de Montgomery.

\begin{abstract}
Stent is a device that is implanted in the trachea, carina or main bronchus with the objective to provide a support that allows maintain the permeability of these structures. There are two groups in agreement with their constitution: Metallic and Silicone stent. Each group has particular properties and indications that must be always considered in individual patient. Metallic stent constitute a very attractive option, due to relative ease in their placement. This has contributed to increase its use in the last years. The accumulated experience in such therapy, has allowed to identify great amount of complications to take into consideration; mainly, in patients who present benign stenosis. These series of comunications at world wide level, has produced recommendations of governmental agencies of other countries; how is the case of FDA, that recommended do not use metallic stents, covered and not covered in benign tracheobronchial stenosis.

We report a case of 59-year-old patient with post intubation tracheal stenosis who was treated in successful form by the placement of Montgomery tube first and after, a Dumon stent.
\end{abstract}

Keywords: tracheal stenosis, Dumon stent, Montgomery tube.

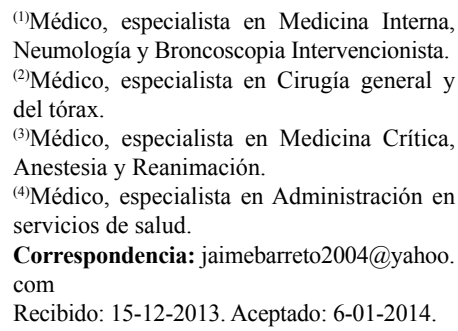

${ }^{(1)}$ Médico, especialista en Medicina Interna, Neumología y Broncoscopia Intervencionista. ${ }^{(2)}$ Médico, especialista en Cirugía general y del tórax.

${ }^{(3)}$ Médico, especialista en Medicina Crítica, Anestesia y Reanimación.

${ }^{(4)}$ Médico, especialista en Administración en servicios de salud.

Correspondencia: jaimebarreto2004@yahoo. com

Recibido: 15-12-2013. Aceptado: 6-01-2014. 


\section{Reporte de caso}

Paciente de 59 años, con antecedente de intubación orotraqueal y ventilación mecánica prolongada por neumonía severa adquirida en comunidad en diciembre de 2009, quien evolucionó con complicaciones en la unidad de cuidado intensivo: shock séptico y sepsis por hongos. Por inestabilidad hemodinámica sólo pudo hacerse traqueostomía hasta el décimo día de intubación. Finalmente se recuperó de su enfermedad y se le dio egreso de UCI sin inconvenientes. Diez días después se le realizó decanulación de traqueostomía sin complicaciones. En los siguientes tres meses empezó a presentar disnea progresiva hasta clase funcional III y disfonía. Se diagnosticó obstrucción de la vía aérea por estenosis subglótica del $50 \%$ de la luz y $5 \mathrm{~cm}$ de longitud. Se realizó cirugía de resección traqueal del segmento comprometido. Evolucionó en forma satisfactoria en el postoperatorio inmediato y se observaron resultados adecuados en el control endoscópico efectuado pocos días después del evento (figura 1). Sin embargo, dos meses después acudió al servicio de urgencias por insuficiencia respiratoria aguda que requirió traqueostomía de emergencia. Posteriormente se hizo una nueva broncoscopia en la que se halló granuloma en la comisura anterior de la glotis y un área de estenosis concéntrica de $2 \mathrm{~cm}$ de longitud a nivel de la anastomosis que ocluía la luz traqueal en un 80\% (figura 2). Se realizó broncoscopia rigida (figura 3) con resección mecánica y electrocirugía de las lesiones descritas y en el mismo procedimiento, a través del estoma de traqueotomía y en conjunto con cirugía de tórax, se colocó un tubo de Montgomery (figura 4), el cual permaneció durante 12 meses sin complicaciones. Un año después se

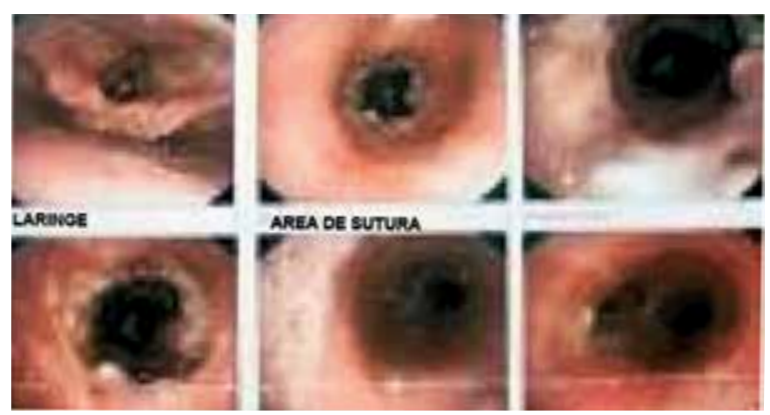

Figura 1. Broncoscopia post-resección traqueal.

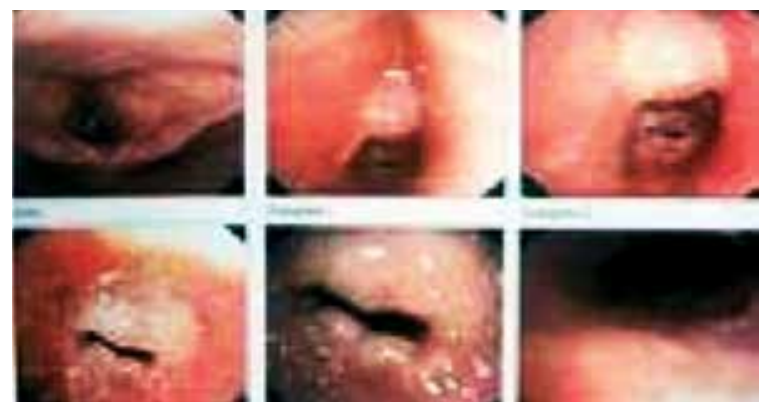

Figura 2. Estenosis postoperatoria.

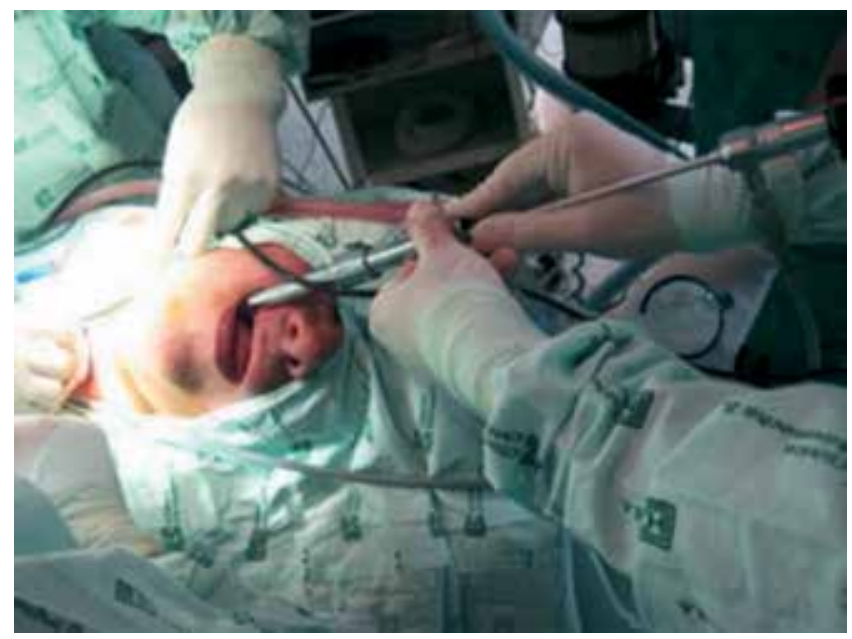

Figura 3. Broncoscopia rígida.

sustituyó este se sustituyó por un stent traqueal de Dumon modelo estenótico 14-12-14 x 40 mm (figura 5), el cual permaneció en posición durante dos años (figura 6), al cabo de los cuales el paciente presentó disnea progresiva, tos y expectoración. Una broncoscopia diagnóstica mostró migración distal al bronquio principal derecho y resolución completa de la estenosis traqueal, razón que ameritó el retiro del stent traqueal vía broncoscopia rígida sin complicaciones (figura 7). En la actualidad permanece sin disnea, sin estenosis subglótica residual, con disfonía leve secundaria a parálisis de la cuerda vocal izquierda.

\section{Discusión}

Los stent traqueobronquiales se usan en general para tratar lesiones obstructivas de etiología maligna ubicadas en las vías aéreas centrales: tráquea, carina y grandes bronquios, por lo general, en conjunto con 


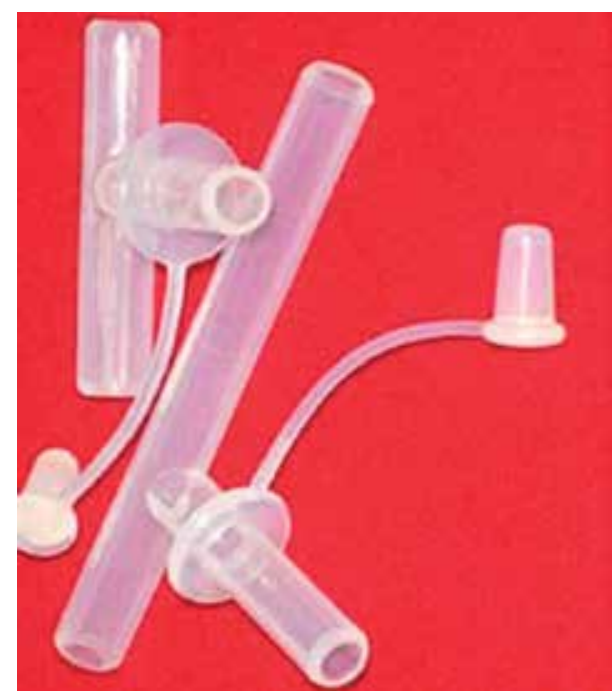

Figura 4. Tubo de Montgomery (cortesía Stening. Argentina).

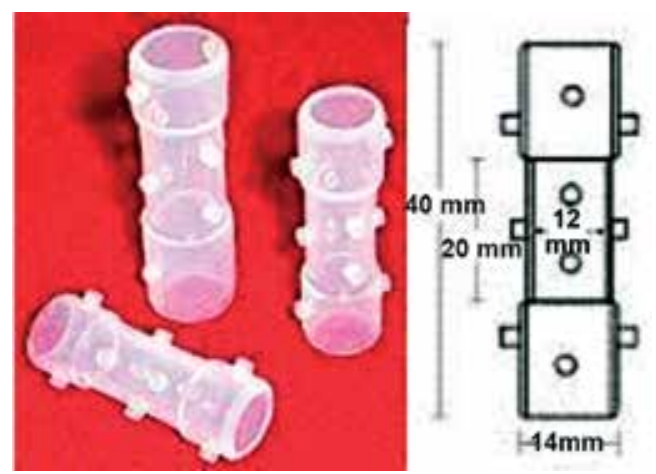

Figura 5. Stent de Dumon estenótico anti-migración (cortesía Stening. Argentina).

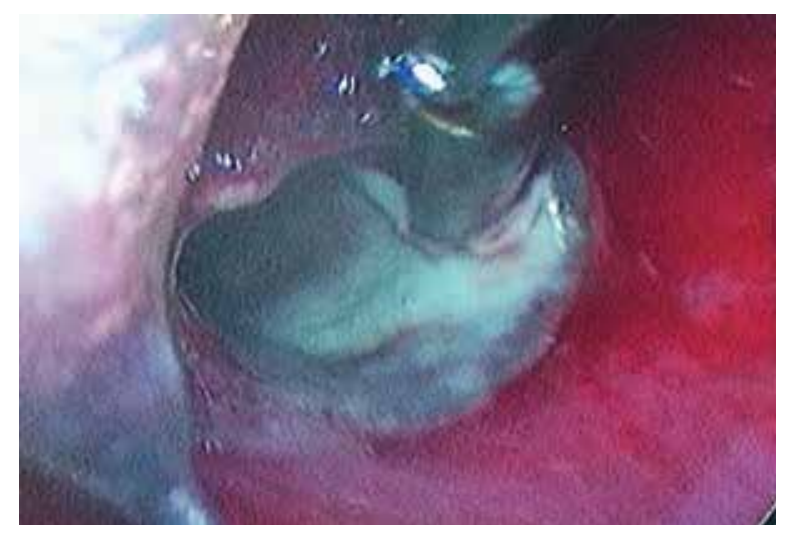

Figura 6. Stent de Dumon en posición.

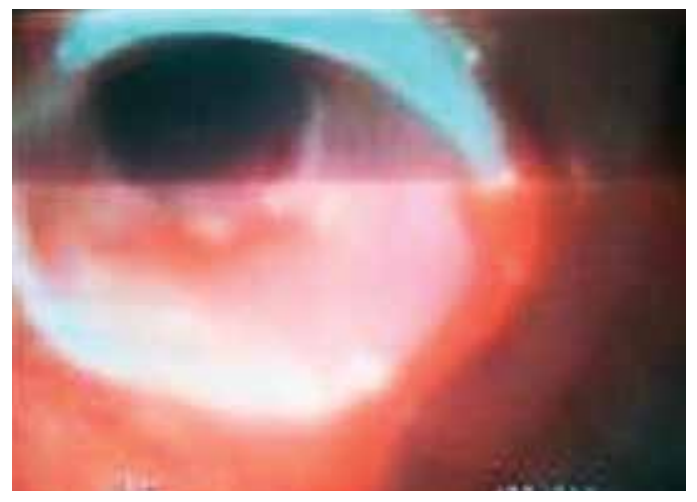

Figura 7. Retirada del stent de Dumon.

otros tratamientos endoscópicos como resección mecánica, láser, electrocirugía, crioterapia, radioterapia endobronquial o terapia fotodinámica. Estas estructuras tubulares sirven para restablecer la permeabilidad de vías aéreas obstruidas y en algunos casos también para sellar fistulas aerodigestivas de etiología benigna o maligna (1).

Los stent de vía aérea son una alternativa de segunda línea para el tratamiento de la estenosis subglótica y traqueal post intubación. Su uso se limita a pacientes no operables, ya sea por razones anatómicas relacionadas con la extensión de la obstrucción o por alteraciones funcionales en su estado de salud que limitan la cirugía de resección traqueal (2).

Hoy se dispone de prótesis de silicona (Dumon) y de prótesis metálicas de diferentes niveles de desarrollo tecnológico, cuya experiencia alcanza casi los veinte años, por lo que pueden hacerse ya algunas observaciones derivadas del uso de ambos sistemas (3).

Los stent metálicos actuales son dispositivos que se adaptan fácilmente al contorno de la vía aérea, resisten muy bien las fuerzas de compresión, se incorporan al epitelio bronquial en las siguientes cuatro semanas a su colocación y alteran menos el movimiento mucociliar. Sin embargo, esta propiedad de incluirse fácilmente en la mucosa se relaciona con su proclividad a desarrollar tejido de granulación a través de la malla metálica y especialmente en los extremos proximal y distal, en donde se observa el mayor grado de fricción con la mucosa traqueobronquial (4). 
La nueva estenosis ocasionada es de tratamiento más complejo que la anterior. El retiro endoscópico del stent en estas condiciones necesariamente implica el uso del broncoscopio rígido $(5,6)$. Este es un procedimiento difícil y riesgoso, que no siempre es posible, de modo que el paciente en esta situación podría verse privado de recibir otros tratamientos de mayor beneficio (stent de silicona y cirugía de resección traqueal). Estas complicaciones se han observado en gran número de reporte de casos en el mundo $(7,8)$ pero no se conocía ninguna serie de casos que pudiera dar soporte a un mayor nivel de evidencia acerca de esta situación. Recientemente, el grupo de neumología intervencionista de la Mayo Clinic, publicó su experiencia en complicaciones de stents metálicos en patologías benignas diversas en el grupo de 28 pacientes que trataron desde 1994 hasta 2006; 35 de 38 stent metálicos debieron ser extraídos por múltiples complicaciones que incluyeron granulación (57\%), migración (25\%), fractura del stent $(8 \%)$, obstrucción dinámica $(8 \%)$ e infección persistente $(4 \%)(9)$.

El tratamiento con stent de Dumon puede ser curativo en cerca de la mitad de los casos como el descrito y en el resto constituye una terapia paliativa aceptable para la obstrucción.

La aparición de nuevos síntomas respiratorios, como en este caso, es indicación de una nueva endoscopia respiratoria para evaluar complicaciones. Es importante anotar que es fundamental evaluar la posibilidad de curación alrededor de los dieciocho meses del tratamiento y si persiste la obstrucción debe sustituirse la prótesis. En el caso expuesto el stent permaneció más tiempo de lo recomendado por razones de orden administrativo y es posible atribuir la migración tardía del mismo al deterioro del dispositivo por tiempo intra traqueal prolongado (10).

La FDA ha recomendado no usar stent metálicos recubiertos y no recubiertos en patologías benignas. Esta recomendación no incluye a los stent de silicona (Dumon), ni a los tubos de Montgomery que actualmente se consideran como el tratamiento endoscópico preferido para pacientes con estenosis secundaria a patología benigna porque son artefactos de fácil extracción (incluso años después de su colocación) y que además tienen menor potencial para inducir tejido de granulación (11).

\section{Conclusión}

La Broncoscopia Intervencionista, con toda su variedad de subprocedimientos, es una alternativa muy valiosa para el tratamiento de pacientes que de otra forma no tendrían una posibilidad de solución satisfactoria al problema de la insuficiencia respiratoria por obstrucción de la tráquea o bronquios principales, específicamente de etiología maligna, aunque también es una opción en estenosis subglótica como terapia complementaria a la cirugía de resección traqueal en situaciones difíciles o como tratamiento de primera línea en casos seleccionados.

Situaciones que anteriormente eran vistas como insalvables, ahora pueden ser tratadas en forma exitosa por equipos de trabajo especializados.

Es importante difundir en Colombia este tipo de tratamientos y orientar a los administradores de salud sobre las indicaciones de este tipo de procedimientos.

\section{Bibliografía}

1. Scuurmans M, Bolliger CT. Silicone airway stents. In: Beamis John F, Mathur Praveen, Mehta Atul, eds. Interventional Pulmonary Medicine. 2004;189:215-238.

2. Ernst A, Feller-Kopman D, Becker H, Mehta A. Central airway obstruction. American Journal of Respiratory and Critical Care Medicine. 2004; 169:1278-1297.

3. Mehta DA. Airway stents. Clin Chest Med. 1999;20:139-151.

4. Dineen K, Jantz M, Silvestri G. Tracheobronchial stents. J Bronchology. 2002;9:127-137.

5. Noppen M, Stratakos G, D'Haese J. Removal of covered self-expandable metallic airway stents in benign disorders. Indications, technique and outcomes. Chest. 2005;127:482-487.

6. Murthy S, Gildea T, Mehta A. Removal of self-expandable metallic stents: is it possible? Sem in Resp and Crit Care Med. 2004;25:381-386.

7. Zakaluzny S, Lane D, Mair E. Complications of tracheobronchial airway stents. Otolaryngology- Head and Neck Surgery. 2003;128:478-488.

8. Peláez M, Cañas A. Reporte de un caso: Estenosis traqueal intrastent: Manejo endoscópico y resección quirúrgica. Rev Colomb Neumol. 2006;18(3):76-79.

9. Swanson K, Edell E, Prakash U B.S, et al. Complications of metal stent therapy in benign airway obstruction. _J Bronchology. 2007;14:90-94.

10. Barreto J. Broncoscopia Intervencionista: una opción a nuestro alcance. Rev Colomb Neumol. 2007;19(4):108-118.

11. FDA Public Health Notification: complications from metallic tracheal stents in patients with benign airway disorders. Disponible en: http:// www.fda.gov/cdrh/safety/072905-tracheal.html. 